\title{
Vitamin D3 promotes oligodendrogenesis and modulates synucleinopathy in lead-induced nigral pars compacta neurotoxicity in rats
}

\author{
R.A. Imam¹, M.R. Abdel-Hamed² \\ ${ }^{1}$ Department of Anatomy and Embryology, Faculty of Medicine, Cairo University, Egypt \\ ${ }^{2}$ Department of Anatomy and Embryology, Faculty of Medicine, Ain Shams University, Egypt \\ [Received: 31 July 2021; Accepted: 29 December 2021; Early publication date: 17 January 2022]
}

\begin{abstract}
Background: Lead-induced neurotoxicity was marked with locomotor and Parkinsonian-like changes. Oligodendrocytes and synucleinopathy were signed to in the pathophysiology of some neurodegenerative diseases. Vitamin D3's (D3) role in substantia nigra pars compacta (SNpc) disorders is debated between neuroscientists. The aim of the study was to investigate lead-induced SNpc neurotoxic changes and explore the possible neuroprotective role of D3 and the possible involvement of oligodendrocytes and $\alpha$-synuclein.

Materials and methods: This study included 40 adult Wistar rats assigned into four equal groups: control, lead (Pb) (in drinking water, 1,000 mg/L), Pb + D3 (D3 injection, 1,000 IU/kg IM; 3 days/week), and D3. After 8 weeks, the rats were sacrificed, and their midbrain underwent biochemical and immunoblotting analysis. Midbrain paraffin blocks were stained for histological and immunohistochemical assessment.

Results: Lead $(\mathrm{Pb})$ had increased significantly $(p<0.05)$ nigral $\alpha$-synuclein and caspase-11 by immunoblotting analysis. Histologically, it induced neurodegeneration in SNpc and significantly decreased neuronal cell density by cresyl violet staining. $\mathrm{Pb}$ also significantly reduced SNpc tyrosine hydroxylase immunoreaction, significantly elevated glial fibrillatory acid protein (GFAP) and $\alpha$-synuclein immunoreaction associated with a mild but significant increase in caspase-3. In the $P b+D 3$ group, all the previous deleterious changes were significantly alleviated in addition to significant upregulation of anti-oligodendrocytes immunoexpression. Conclusions: Lead $(\mathrm{Pb})$ may induce SNpc neurotoxicity presumably via activation of caspase-11 and $\alpha$-synuclein. D3 may modulate this neurotoxicity probably through an oligodendrogenic effect. (Folia Morphol 2023; 82, 1: 42-52)
\end{abstract}

Key words: lead $(\mathrm{Pb})$, pars compacta, D3-synucleiopathy, oligodendrocytes

\section{INTRODUCTION}

As an environmentally toxic heavy metal, lead $(\mathrm{Pb})$ induced profound clinical changes in most body systems, particularly on the nervous system (the primary target of the $\mathrm{Pb}$ ) [1]. It had been evidenced that frequent exposure to lead is linked with Parkinsonism-like motor deficits, suggesting strong evidence for a role of $\mathrm{Pb}$ in the development of Parkinson's disease (PD)

Address for correspondence: Dr. R.A. Imam, Department of Anatomy and Embryology, Faculty of Medicine, Cairo University, Cairo, Egypt tel: +2001006114696; e-mail: redaabdelnasser@cu.edu.eg; abdelnasserreada@gmail.com

This article is available in open access under Creative Common Attribution-Non-Commercial-No Derivatives 4.0 International (CC BY-NC-ND 4.0) license, allowing to download articles and share them with others as long as they credit the authors and the publisher, but without permission to change them in any way or use them commercially. 
(the most typical neurobiological motion disorder of old age predominating in $2-3 \%$ of $\geq 65$ years old worldwide) $[14,18]$. Oligodendrocytes, myelin producers of the central nervous system, are involved in the pathophysiology of some neurodegenerative diseases [17]. For example, aggregates of $\alpha$-synuclein in oligodendrocytes, forming glial cytoplasmic inclusions, were a main neuropathological finding in multiple system atrophy neurodegenerative disease [13]. However, the role of oligodendrocytes in pars compacta neurodegenerative disorders is not yet clear. Inclusion synucleinopathy is a particular pathological feature for some neuronal disorders [21]. Caspase-11 had been early reported to mediate nigral neurotoxicity in 1-Methyl-4-Phenyl-1, 2, 3, 6-Tetrahydropyridine (MPTP)-received mice [7]. Vitamin D3 had been proved to protect against lead-induced adverse changes in some organs like kidneys and testis [4]. However, neuro researchers debate the vitamin D3 role in substantia nigra pars compacta (SNpc) neurodegenerative disorders [6]. Some authors have reported that D3 may protect SNpc neurons against oxidative stress and inflammation in some neuronal conditions [8]. This study aimed to investigate lead-induced SNpc neurotoxicity, explore its relation with $\alpha$-synuclein, oligodendrocytes and caspases-3, -11 , and explore the possible protective role of $\mathrm{D} 3$ against this neurotoxicity.

\section{MATERIALS AND METHODS}

\section{Materials}

Lead acetate was purchased in powder form from El-Gomhouria Company (Cairo, Egypt). D3 was supplied in injection (Devarol S 200,000 IU, 2 mL ampoule; Egypt Memphis Company, Cairo, Egypt). Vitamin D3 kits for serum D3 were purchased as Elisa kits from MyBioSource, USA. ReadyPrepTM protein extraction kit was supplied by Bio-Rad Laboratories Inc. (Hercules, CA, USA). The quantitative protein analysis Bradford Protein Assay Kit was provided by Bio Basic Inc. (Markham, ON, Canada). Polyacrylamide gels were extracted using TGX Stain-Free ${ }^{T M}$ FastCast $^{T M}$ Acrylamide Kit purchased from Bio-Rad Laboratories Inc. Caspase 11 primary antibody (caspase-11; Rat mAb) was purchased from Cell Signaling Technology (Beverly, MA, USA). $\alpha$-synuclein primary antibody for immunoblotting analysis was purchased from Santa Cruz Biotechnology (Santa Cruz, CA, USA). Immunoblotting technique horseradish peroxidase (HRP)-conjugated secondary antibody was goat anti-rabbit IgG-HRP-1 mg Goat mab (Novus Biologicals, Littleton, CO, USA). The chemiluminescent substrate was ClarityTM Western ECL Substrate (Bio-Rad Laboratories Inc.). Primary antibodies utilised in immunohistochemistry were anti-oligodendrocytes (Olig-2) rabbit polyclonal (GeneTex, Inc., Irvine, CA, USA), glial fibrillatory acid protein (GFAP) and anti-D3 were rabbit polyclonal (Dako, Denmark), tyrosine hydroxylase $(\mathrm{TH})$ and $\alpha$-synuclein were rabbit polyclonal (Abcam, UK), caspase-3 was rabbit, polyclonal (Santa Cruz Biotechnology, USA).

\section{Animals}

The present study strictly adhered to animal research ethics and was approved by CU-IACUC (no. III-F-47-20). Forty adult Wistar rats weighing 150-200 g were assigned into four equal groups and housed at the animal house of the Faculty of Medicine, Cairo University, Cairo, Egypt. They were subjected to standard animal conditions 2 weeks before the experiment for acclimatisation, fed ad libitum, and grouped into the control group (I) received intramuscular saline, $\mathrm{Pb}$ group (II) received a lead in drinking water, $\mathrm{Pb}+\mathrm{D} 3$ group (III) received lead concomitant with D3 injection and D3 group (IV) received D3 injection.

\section{Chemicals}

Lead. The powder was dissolved in drinking water and given to the rats at a dosage of $1,000 \mathrm{mg} / \mathrm{L}$ [4].

Vitamin D3. The vitamin was given at a dosage of $1,000 \mathrm{IU} / \mathrm{kg}$ IM (3 days/week) [4].

All rats were weighed, blood samples were obtained to measure serum D3 by Elisa and serum lead by atomic absorption spectrophotometer (model Sen$s A A$, Australia). The rats were sacrificed by guillotines method 8 weeks from the experiment to minimize brain injury. Their brains were obtained and subjected to biochemical and histological investigations.

\section{Biochemical analysis}

Parts from the ventral midbrain were used for biochemical measurement of lead in tissue and immunoblotting analysis (for caspase-11 and $\alpha$-synuclein).

Measurement of lead in ventral midbrain. Air-dried ventral midbrain tissue was digested with $8 \mathrm{~mL}$ of concentrated sulphuric acid then heated for 5 minutes on a hot plate to remove all fumes with the addition of perchloric acid followed by metal analysis by atomic absorption spectrophotometer (model SensAA, Australia). 
Immunoblotting analysis technique. The ReadyPrep ${ }^{\mathrm{TM}}$ protein extraction kit (total protein) was added for each homogenized ventral midbrain tissues sample. A Bradford assay was conducted, followed by polyacrylamide gel electrophoresis loading. Protein bands were transferred from the gel to the membrane using BioRad Trans-Blot Turbo. The membrane was blocked in tris-buffered saline with Tween 20 (TBST) buffer and 3\% bovine serum albumin (BSA) at room temperature for 1 hour. Primary antibodies of caspase-11 and $\alpha$-synuclein were diluted in TBST followed by overnight incubation in each primary antibody solution against the blotted target protein at $4^{\circ} \mathrm{C}$. Incubation was conducted in the HRP-conjugated secondary antibody solution against the blotted target protein for 1 hour at room temperature. The chemiluminescent substrate was applied to the blot as equal volumes from solutions $A$ (Clarity Western Luminal/Enhancer Solution) and B (peroxidase solution). The chemiluminescent signals were captured, and image analysis software was performed to read the band intensity by protein normalisation on the ChemiDoc MP imager.

\section{Histological assessment}

The midbrain was preserved in formalin, processed for paraffin blocking, utilised for histological and immunohistochemical staining. Paraffin blocked sections were processed for staining with haematoxylin and eosin (H\&E) and cresyl violet stain. For cresyl violet staining, tissue sections were soaked in paraformaldehyde, washed into Pbs, soaked with cresyl violet solution, then dehydrated using ascending grades of ethanol $70 \%, 90 \%$, and finally $100 \%$.

\section{Immunohistochemical techniques [3]}

Midbrain sections were deparaffinised, underwent heat-induced epitope retrieval and stained with anti-D3 (to evaluate D3; dilution, 1:100), TH (to evaluate for dopaminergic neurons; dilution, 1:100), GFAP (to evaluate for astrocytic activity; dilution, 1:100), caspase-3 (dilution 1:100) (to assess for apoptosis), $\alpha$-synuclein (to detect synucleinopathy, dilution 1:50) and anti-Olig-2 (to evaluate for oligodendrocytes; dilution, 1:100). The slides were entered into the Dako automated system (EnVision Flex-peroxidase blocked). Moreover, the Dako EnVision Flex/HRP was added as the secondary antibody. Leica ICS150 microscope camera was used to photograph H\&E and immunohistochemical slides.

\section{Histomorphometric measurement}

The area percentage of the positive immune reaction of anti-D3, TH, GFAP, anti-Olig- $2, \alpha$-synuclein, and caspase- 3 was measured using image $J$ analysis software, ten randomly chosen fields measurements ( $\times 400$ magnification) from each group were taken and statistically analysed. Also, neuronal cell density by cresyl violet staining was determined by the number of surviving neurons in each field ( $\times 400$ magnification) as measured by image $\mathrm{J}$ software. According to Wang et al. 2010 [19], non-viable neurons were determined as those with dense cresyl violet staining and pyknotic nuclei.

\section{Ethical approval}

All the study procedures and manipulations were done according to the ARRIVE guidelines and the study was approved by Cairo University Institute of Animal Care and Use Committee (CU-IACUC) under No III-F-47-20.

\section{Statistical analysis}

GraphPad Prism 8 was utilized to analyse all gathered data represented as mean \pm standard error of the mean (SEM). Analysis of variance and Tukey's test were conducted for in-between group comparison with a cut-off $p$-value of $\leq 0.05$ for significance.

\section{RESULTS}

\section{Clinical data}

No mortality had been recorded among rats. The body weight of rats at the end of the experiment in the $\mathrm{Pb}+\mathrm{D} 3$ and D3 groups was significantly increased compared with the control and $\mathrm{Pb}$ groups (Table 1).

\section{Biochemical results}

Lead and D3 measurement in blood. Lead $(\mathrm{Pb})$ in blood had increased significantly in $\mathrm{Pb}$ and $\mathrm{Pb}+\mathrm{D} 3$ groups compared to control and D3 groups. It showed a mild significant decrease in the $\mathrm{Pb}+\mathrm{D} 3$ group compared to the $\mathrm{Pb}$ group (Table 1). D3 in blood had increased significantly in Pb + D3 and D3 groups compared to control and $\mathrm{Pb}$ groups (Table 1).

Lead measurement in the midbrain. Lead $(\mathrm{Pb})$ in the midbrain had increased significantly in $\mathrm{Pb}$ and $\mathrm{Pb}+\mathrm{D} 3$ groups compared to control and D3 groups (Table 1).

Western blot results. Lead $(\mathrm{Pb})$ significantly raised the protein expressions of $\alpha$-synuclein in the anterior part of the midbrain (ventral) compared to the control 
Table 1. Bodyweight (BW) of rats, serum $\mathrm{D} 3$ and $\mathrm{Pb}$, midbrain $\mathrm{Pb}$ level, and cell density of substantia nigra pars compacta (SNpc) neurons by cresyl violet among different groups

\begin{tabular}{lccccc}
\hline Groups & $\begin{array}{c}\text { BW } \\
{[\mathbf{g}]}\end{array}$ & $\begin{array}{c}\text { Serum Pb } \\
{[\mu \mathrm{g} / \mathrm{dL}]}\end{array}$ & $\begin{array}{c}\text { Serum D3 } \\
{[\mathbf{n g} / \mathbf{d L}]}\end{array}$ & $\begin{array}{c}\text { Midbrain Pb level } \\
{[\mu \mathrm{g} / \mathbf{m L}]}\end{array}$ & $\begin{array}{c}\text { The cell density of SNpc } \\
\text { neurons by cresyl violet }\end{array}$ \\
\hline Control & $222 \pm 3.62$ & $3.2 \pm 0.26$ & $26.42 \pm 3.40$ & $0.1093 \pm 0.021$ & $47.7 \pm 2.35$ \\
Lead $(\mathrm{Pb})$ & $187 \pm 4.32^{\mathrm{a}}$ & $50.9 \pm 2.73^{\mathrm{a}}$ & $20.63 \pm 3.02$ & $2.33 \pm 0.192^{\mathrm{a}}$ & $23.80 \pm 2.30^{\mathrm{a}}$ \\
$\mathrm{Pb}+\mathrm{D3}$ & $280 \pm 7.06^{\mathrm{a}, \mathrm{b}}$ & $39.59 \pm 3.44^{\mathrm{a}, \mathrm{b}}$ & $77.35 \pm 3.81^{\mathrm{a}, \mathrm{b}}$ & $1.751 \pm 0.364^{\mathrm{a}}$ & $34.70 \pm 2.18^{\mathrm{a}, \mathrm{b}}$ \\
Vitamin D3 & $279.8 \pm 7.31^{\mathrm{a}, \mathrm{b}}$ & $3.08 \pm 0.40^{\mathrm{b}, \mathrm{c}}$ & $82.93 \pm 4.36^{\mathrm{a}, \mathrm{b}}$ & $0.1391 \pm 0.030^{\mathrm{b}, \mathrm{c}}$ & $46.20 \pm 2.66^{\mathrm{b}, \mathrm{c}}$ \\
\hline
\end{tabular}

${ }^{a} \mathrm{p}<0.05$ i.e. statistically significant when compared with the control group; ${ }^{\mathrm{b}} \mathrm{p}<0.05$ when compared with the $\mathrm{Pb}$ group; ${ }^{\mathrm{c}} \mathrm{p}<0.05$ when compared with $\mathrm{Pb}+\mathrm{D} 3$ group (data are represented as mean \pm standard error of the mean)

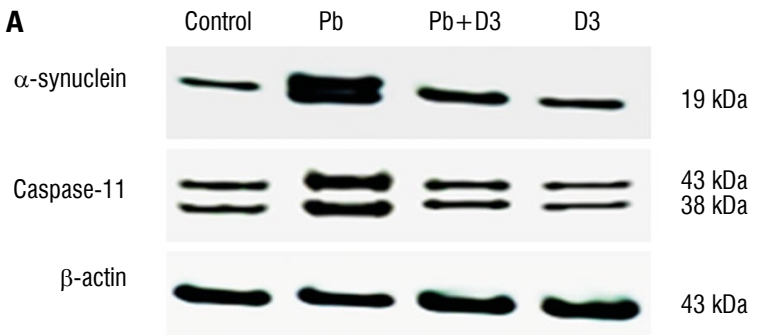

B

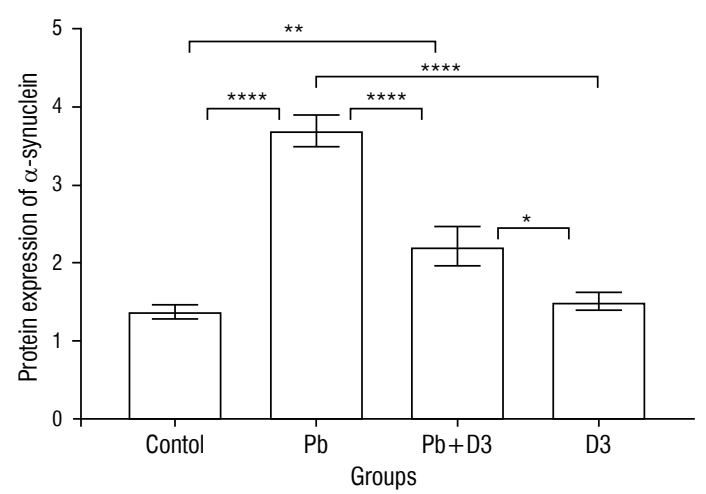

C

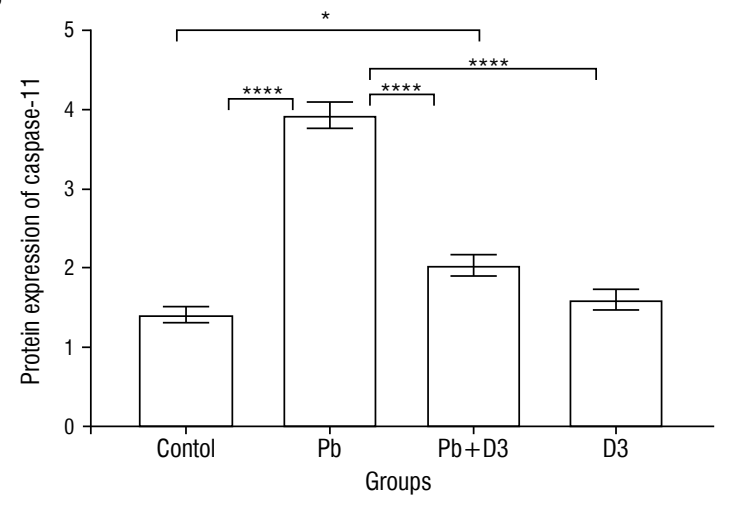

Figure 1. A. Western blot analysis of protein expression of $\alpha$-synuclein and caspase-11 among the different groups with $\beta$-actin as the internal standard. B, C. The two graphs show that lead $(\mathrm{Pb})$ had significantly increased $\alpha$-synuclein and caspase-11 compared with control. In the $\mathrm{Pb}+\mathrm{D} 3$ group, both proteins were significantly decreased compared with the $\mathrm{Pb}$ group; * statisticalIy significant when $\mathrm{p}<0.05 ;{ }^{* *}$ when $\mathrm{p}<0.01 ;{ }^{* * *}$ when $\mathrm{p}<0.001 ;{ }^{* * *}$ when $\mathrm{p}<0.0001$ (error bar $=$ standard error of the mean). and D3 groups (2.68- and 2.45-fold, respectively). The same was true for caspase-11 in the Pb group compared with the control and D3 groups (2.8- and 2.4-fold, respectively) via Western blot analysis. Compared with the lead group, the simultaneous addition of D3 to lead in group III significantly decreased $\alpha$-synuclein and caspase- 11 ( $66 \%$ and $92 \%$, respectively). However, they were still significantly elevated ( $60 \%$ and $43 \%$ for $\alpha$-synuclein and caspase-11, respectively) compared with the control group (Fig. 1).

\section{Morphological results}

The control group, SNpc typical neurons via H\&E stain (Fig. 2), appeared with vesicular nuclei and lightly stained cytoplasm. Pb group SNpc exhibited interstitial oedema, darkly stained neurons with pyknotic nuclei, degenerated neurons (karyolysis), lost neurons, and infiltrated glial cells. A few SNpc neurons in the $\mathrm{Pb}+\mathrm{D} 3$ group were darkly stained, whereas most neurons were preserved. D3 group SNpc exhibited typically appearing neurons.

Via cresyl violet staining (Fig. 3), the control group exhibited standard staining of the nuclei and cytoplasm while $\mathrm{Pb}$ group SNpc showed few neurons with dark cresyl violet staining. A few SNpc neurons in the $\mathrm{Pb}+\mathrm{D} 3$ group were densely stained, whereas most neurons were preserved. The D3 group SNpc exhibited standardly stained neurons. The cell density of SNpc by cresyl violet staining in histomorphometric analysis revealed a significant decrease in the number of viable neurons in the $\mathrm{Pb}$ group compared to the control and D3 group ( $50 \%, 48 \%$, respectively). The $\mathrm{Pb}+\mathrm{D} 3$ group showed a significant increase (45\%) in the number of viable neurons than the $\mathrm{Pb}$ group (Table 1).

\section{Immunohistochemical results}

The control and lead SNpc groups exhibited scanty anti-D3 immunostaining (Fig. 4). The Pb + D3 

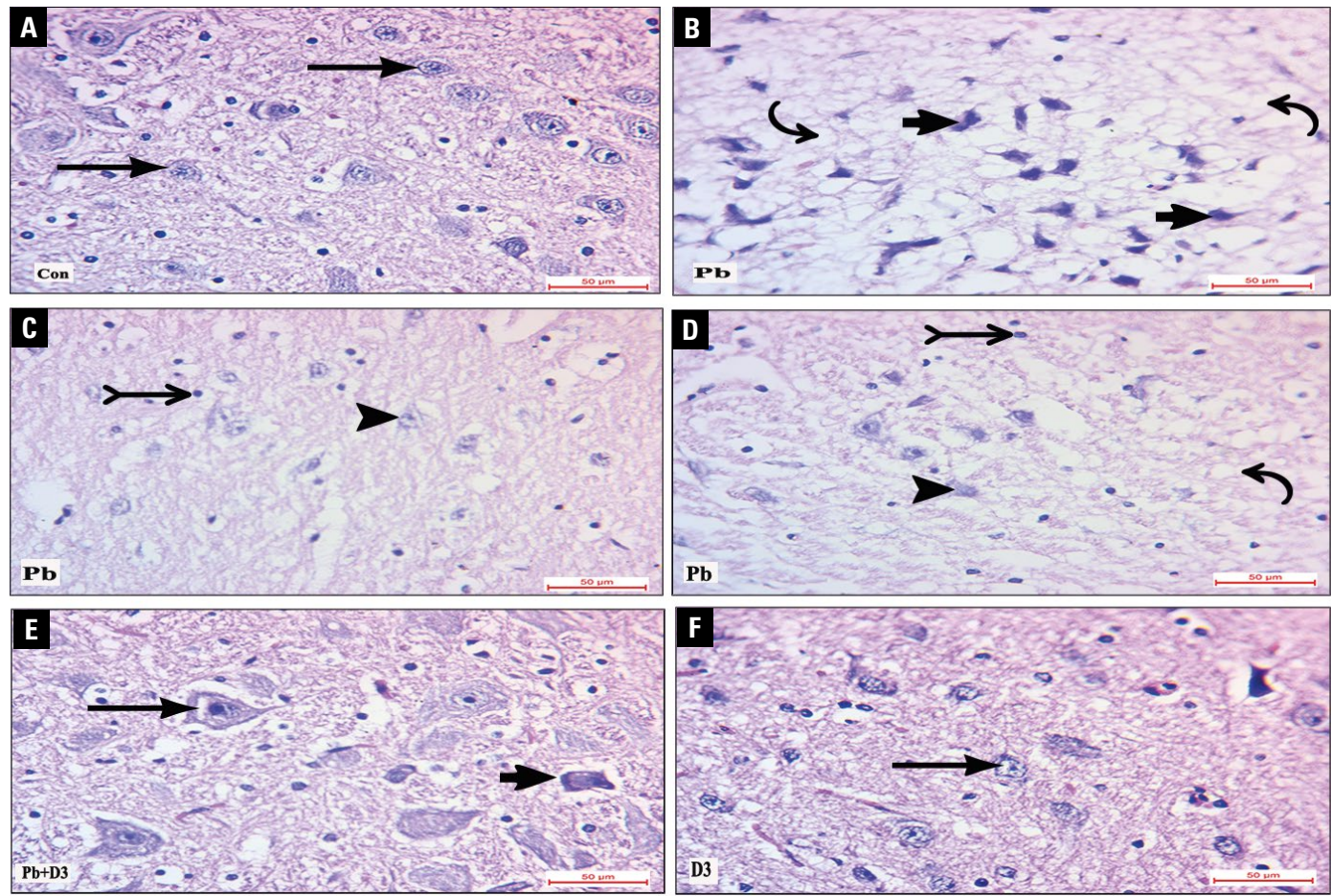

Figure 2. A. The control group (Con) substantia nigra pars compacta (SNpc) exhibits typical neurons with vesicular nuclei and lightly stained cytoplasm (arrows); B, C, D. The lead (Pb) group SNpc with interstitial oedema (curved arrows), darkly stained neurons with pyknotic nuclei (incomplete arrows), degenerated neurons (karyolysis) (arrowheads) and infiltrated glial cells (tailed arrows); E. A few SNpc neurons in the $\mathrm{Pb}+\mathrm{D} 3$ group are darkly stained, whereas most neurones are preserved; F. Vitamin D3 SNpc group displays typically appearing neurons $(\mathrm{H} \& \mathrm{E} \times 400$, scale bar $=50 \mu \mathrm{m})$.
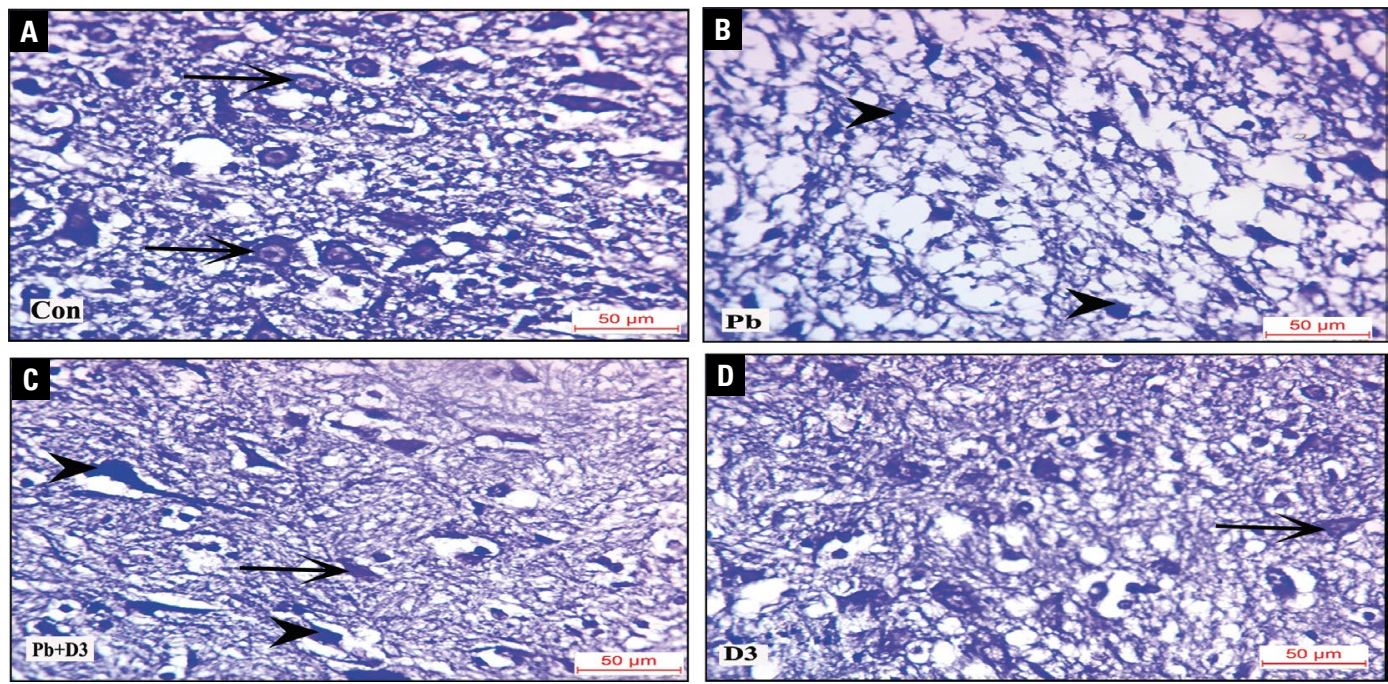

Figure 3. A. The control group (Con) substantia nigra pars compacta ( $\mathrm{SNpc}$ ) with neurons with standard cresyl violet staining of the nuclei and cytoplasm (arrows); B. The lead ( $\mathrm{Pb}$ ) group SNpc shows degenerated neurons with dense cresyl violet staining and pyknotic nuclei (arrowheads); C. A few SNpc neurons in the Pb + D3 group are densely stained, whereas most neurones are preserved; D. The D3 group SNpc with standardly stained neurons (cresyl violet $\times 400$, scale bar $=50 \mu \mathrm{m}$ ).

and D3 groups showed strong anti-D3 immunostaining. Histomorphometric data revealed that the area percentage of positive anti-D3 immunoreaction in the $\mathrm{Pb}+\mathrm{D} 3$ group significantly increased compared with both control and $\mathrm{Pb}$ groups (11.05and 12.45 -fold, respectively). Additionally, in the D3 group, the area percentage of the positive anti-D3 significantly increased compared with both control 

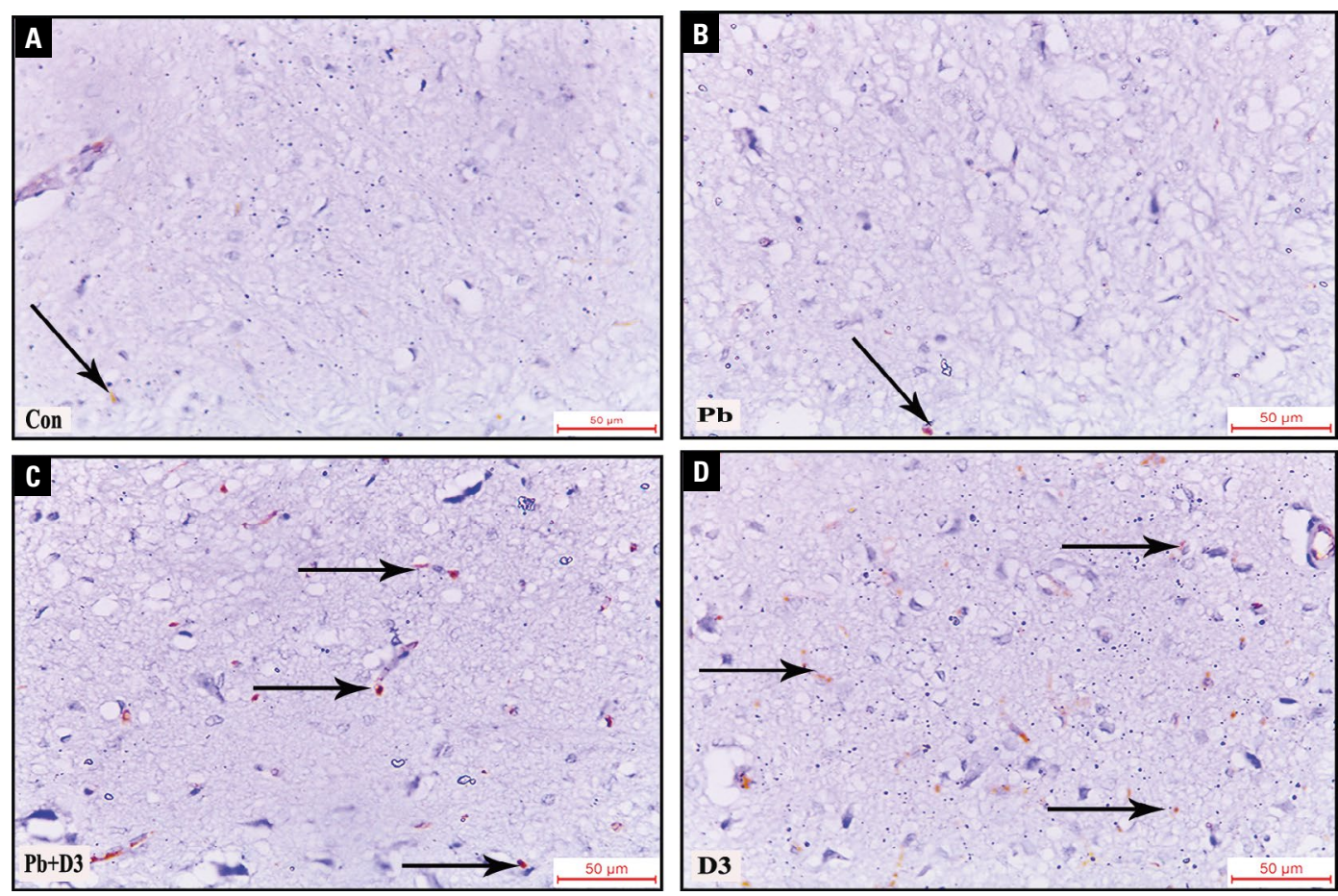

Figure 4. A, B. The control and lead groups respectively (Con, $\mathrm{Pb}$ ) substantia nigra pars compacta (SNpc) appear with scanty anti D3 immunostaining, whereas the Pb + D3 (C) and D3 (D) groups appear with increased anti-D3 immunostaining (arrows) (anti-D3 $\times 400, ~ s c a l e ~ b a r ~=50 \mu \mathrm{m}$ ).

and $\mathrm{Pb}$ groups (13.6- and 15.37-fold, respectively; Fig. 5).

Via TH immunostaining (Fig. 6), the control and D3 groups SNpc neurons exhibited a strong TH reaction, whereas the $\mathrm{Pb}$ group SNpc appeared with a few neurons and decreased $\mathrm{TH}$ reactions. The $\mathrm{Pb}+\mathrm{D} 3$ SNpc revealed increased reaction compared with the $\mathrm{Pb}$ group. Histomorphometric data showed that the area per cent of positive TH immune reaction in the $\mathrm{Pb}$ group was significantly decreased compared with control and D3 groups (49\%, 43\%, respectively). In contrast, it was significantly increased in the $\mathrm{Pb}+\mathrm{D} 3$ group compared with the $\mathrm{Pb}$ group (52\%) (Fig. 5).

Via anti-Olig-2 immunostaining (Fig. 7), the control SNpc group appeared with a standard anti-Olig-2 reaction, whereas the lead SNpc group appeared with mildly decreased reaction. The $\mathrm{Pb}+\mathrm{D} 3 \mathrm{SNpc}$ group exhibited increased reaction, whereas the D3 group exhibited mild increased reaction. Via histomorphometric analysis, the area percentage of positive anti-Olig-2 significantly increased in the Pb + D3 group compared with that in the control, $\mathrm{Pb}$, and $\mathrm{D} 3$ groups (1.96-, 2.11-, and 2.13-fold, respectively) (Fig. 5).

Via GFAP immunostaining (Fig. 8), the lead SNpc group exhibited increased reaction, whereas the $\mathrm{Pb}+$ + D3 and D3 groups appeared with relatively normal reactions. Via histomorphometric analysis, the area percentage of the positive GFAP in the Pb group significantly increased compared with the control and D3 groups (3.27- and 2.37-fold, respectively). In contrast, it was significantly decreased (60.6\%) in the $\mathrm{Pb}+\mathrm{D} 3$ group compared with the $\mathrm{Pb}$ group, although it was still significantly increased (1.99-fold) compared with the control group (Fig. 5).

Via caspase-3 immunostaining (Fig. 9), the control and D3 group SNpc appeared with a nearly negative reaction, whereas the $\mathrm{Pb}$ group SNpc appeared with mildly increased reaction. The $\mathrm{Pb}+\mathrm{D} 3$ group SNpc exhibited minimally increased reaction. Via histomorphometric analysis, the area percentage of positive caspase-3 reaction has increased mildly although significantly in the $\mathrm{Pb}$ group compared with the control and D3 (2.3-, 1.8-fold, respectively). It decreased significantly in the $\mathrm{Pb}+\mathrm{D} 3$ group (34\%) compared to the $\mathrm{Pb}$ group (Fig. 5).

Via $\alpha$ synuclein immunostaining (Fig. 10), the control group SNpc showed a nearly negative immune reaction, whereas the lead ( $\mathrm{Pb}$ group) SNpc appeared with markedly increased reaction (arrows). The $\mathrm{Pb}+\mathrm{D} 3$ $\mathrm{SNpc}$ exhibited a reduced reaction than the $\mathrm{Pb}$ group, whereas the D3 group SNpc neurons appeared with scanty $\alpha$-synuclein reaction. Via histomorphometric analysis, the area percentage of positive $\alpha$-synuclein 
A

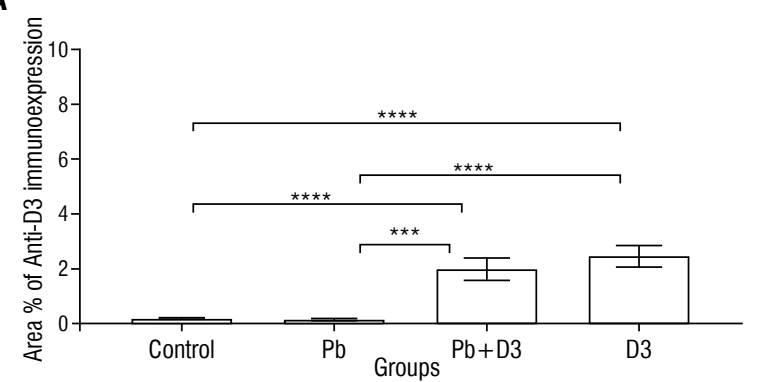

C

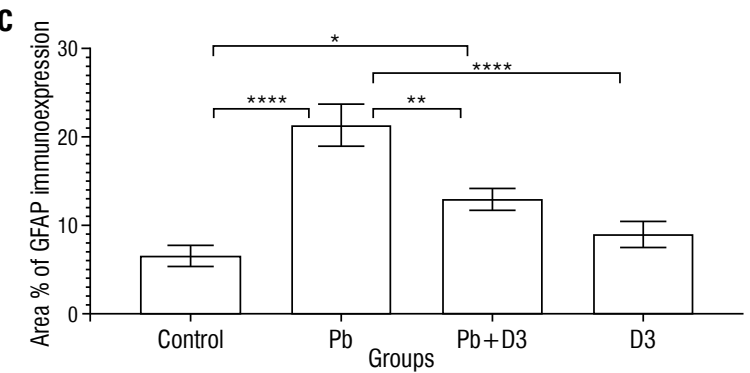

E

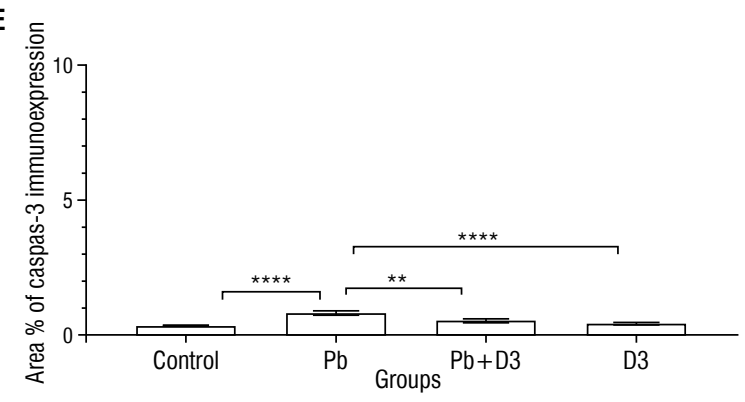

B

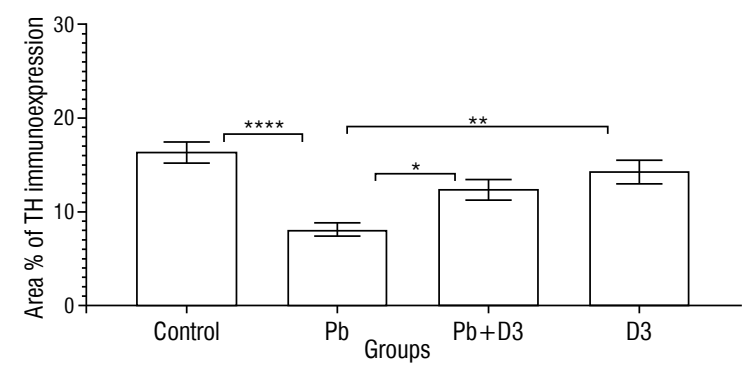

D
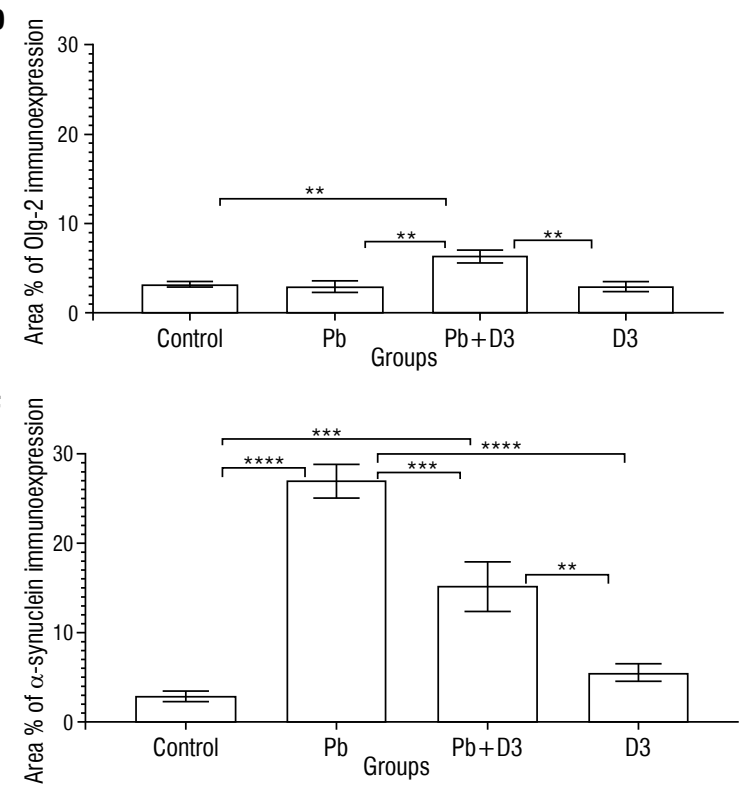

Figure 5. Bar charts of the area percentage of positive anti-D3 (A), tyrosine hydroxylase (TH) (B), glial fibrillatory acid protein (GFAP) (C), oligodendrocytes (Olig-2) (D), caspase-3 (E) and $\alpha$-synuclein (F) immunoreaction in different groups; ${ }^{*}$ statistically significant when $p<0.05$; ${ }^{* *}$ when $\mathrm{p}<0.01 ;{ }^{* * *}$ when $\mathrm{p}<0.001 ;{ }^{* * *}$ when $\mathrm{p}<0.0001$ (error bar $=$ standard error of the mean).

reaction has increased significantly in the $\mathrm{Pb}$ group compared to the control and D3 groups (9.8-, 4.8-fold, respectively). In the $\mathrm{Pb}+\mathrm{D} 3$ group, it was significantly reduced (44\%) as compared to the $\mathrm{Pb}$ group.

\section{DISCUSSION}

In this study, significantly accumulated lead in the ventral midbrain had induced SNpc neurotoxic changes, as evidenced by activation of $\alpha$-synuclein, caspase-3 and caspase-11, GFAP, as well as downregulation of TH. Previously proved, lead deteriorated motor functions in rats and degenerated cerebellar neurons via oxidative stress mechanisms [11]. In this work, Pb had significantly elevated nigral $\alpha$-synuclein levels. Accumulation of misfolded and aggregated $\alpha$-synuclein in Lewy bodies is a main neurobiological feature for PD resulting in disrupting cellular functions and inducing mitochondria injury as well as synaptic dysfunctions [9]. Additionally, nigral caspase-11 and, to a lesser extent, caspase-3 were significantly elevated in the $\mathrm{Pb}$ group in the present study suggesting their potential mediating role in nigral neuronal cell death. Concordantly, it has been reported that MPTP-induced nigral neurotoxicity was mediated through caspase-11 activation [7]. Studies with caspase-11 blocking agents are still needed to confirm the latter finding of the current work. Strategies monitoring and modifying inflammation and cell death were currently suggested to improve the management of PD, and elevated plasma-borne inflammasome NLRP3 was associated with PD status [2]. Further studies are still warranted to elucidate the role of inflammasomes in the lead-induced SNpc neurotoxicity. Biochemically, lead was significantly increased in the serum and midbrain of this study's $\mathrm{Pb}$ group, suggesting its accumulation in SNpc neurons. Morphologically, the accumulated $\mathrm{Pb}$ deteriorated the SNpc dopaminergic neurons, significantly decreased cell density by cresyl violet staining, and upregulated GFAP immunoreaction considerably. Additionally, 

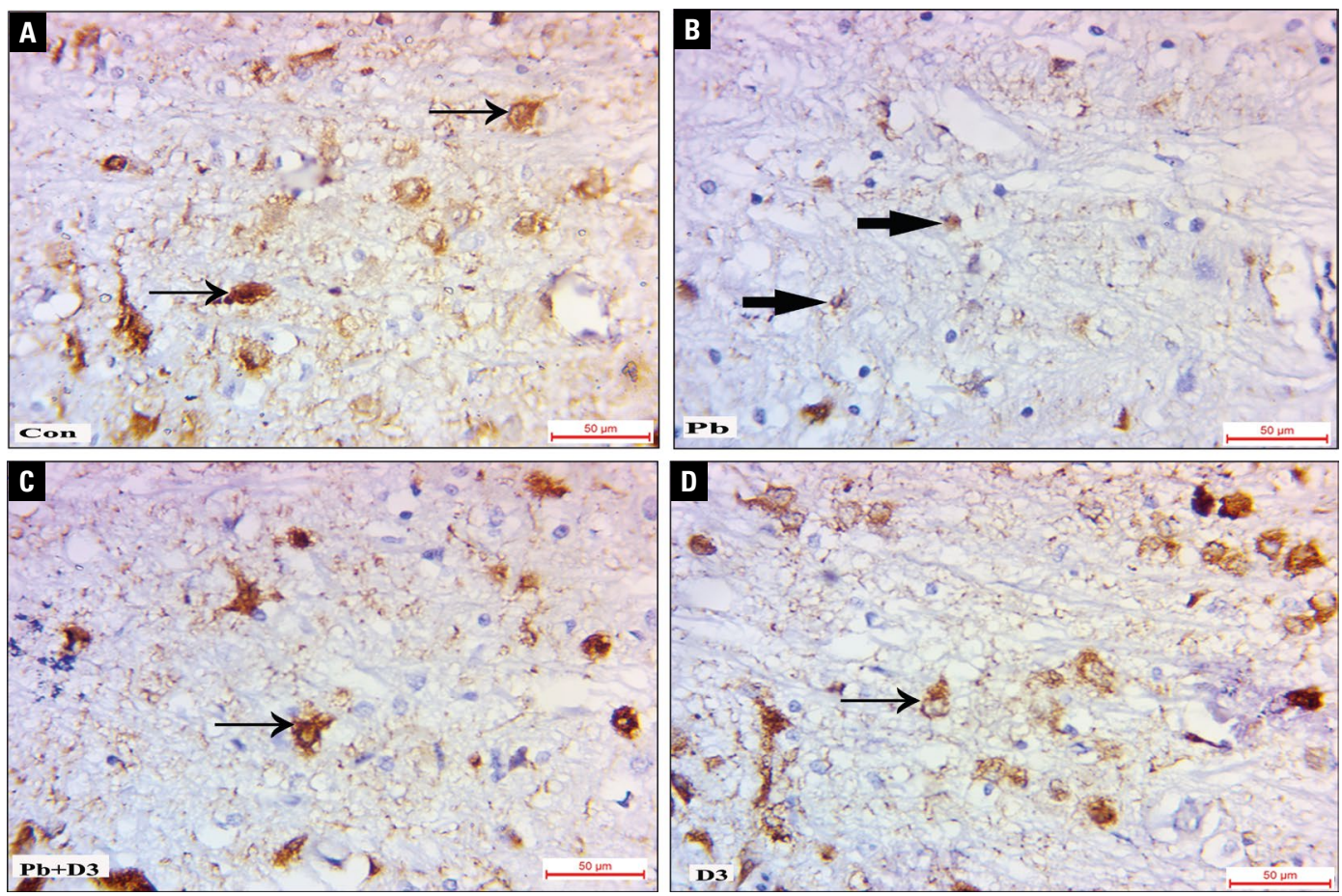

Figure 6. A. The control (Con) group substantia nigra pars compacta ( $\mathrm{SNpc}$ ) neurons exhibit a strong tyrosine hydroxylase (TH) reaction (arrows), whereas lead $(\mathrm{Pb})$ group (B) SNpc appears with few neurons and decreased TH reactions (incomplete arrow); C. The Pb $+\mathrm{D} 3$ SNpc shows an increased reaction than the Pb group; D. Vitamin D3 group SNpc neurons exhibit a strong TH reaction (TH $\times 400$, scale bar $=50 \mu \mathrm{m})$.
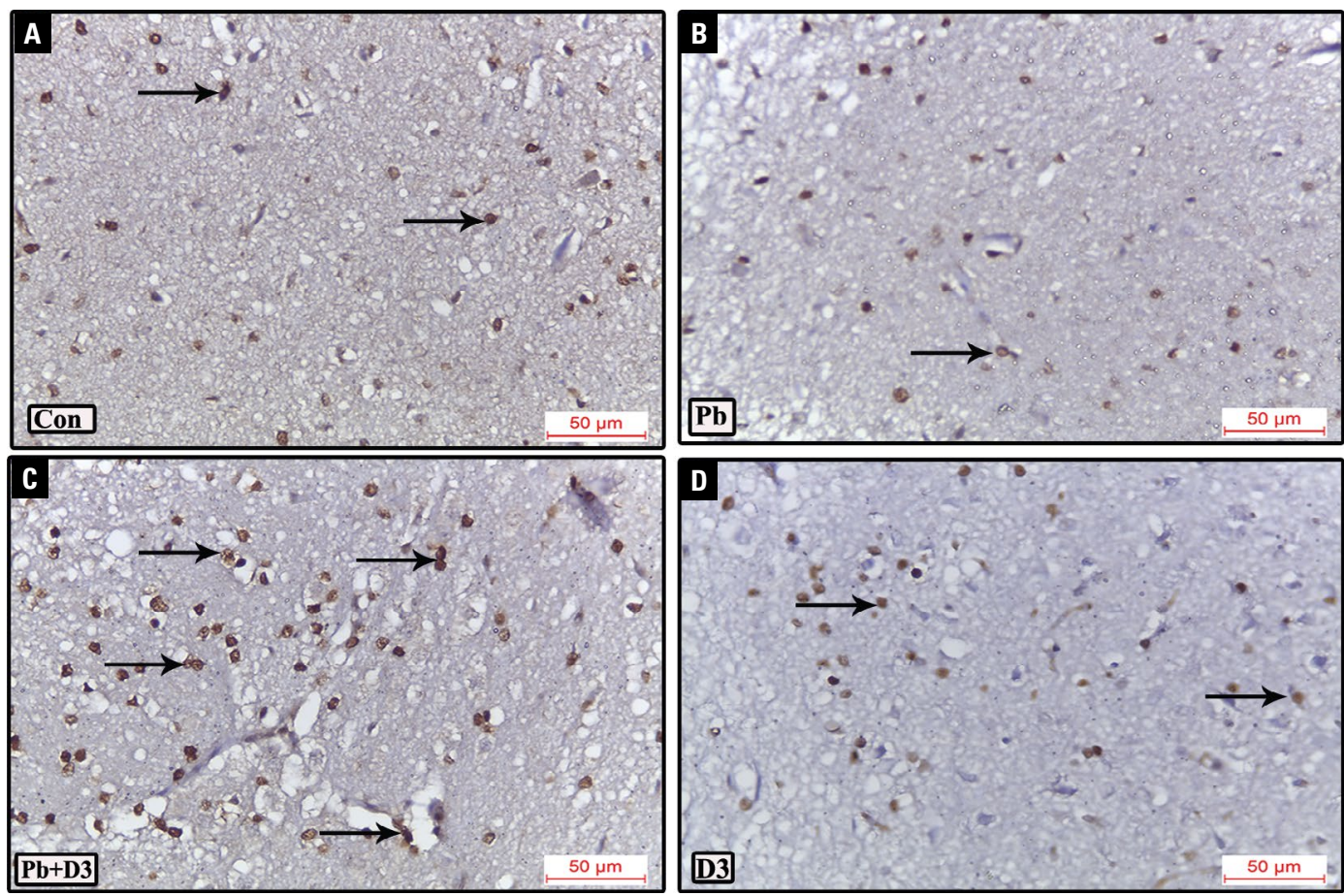

Figure 7. A, B. The control (Con) group substantia nigra pars compacta (SNpc) appears with normal anti-oligodendrocytes (Olig-2) reaction (arrows) while lead $(\mathrm{Pb})$ group SNpc appeared with mildly increased reaction; $\mathbf{C}$. The $\mathrm{Pb}+\mathrm{D} 3$ group SNpc exhibits increased reaction; D. Vitamin D3 group exhibits mild increased reaction (anti-Olig-2 $\times 400$, scale bar $=50 \mu \mathrm{m}$ ).

$\mathrm{Pb}$ significantly reduced the area percentage of $\mathrm{TH}$ immunopositive dopaminergic neurons, denoting its dangerous neurotoxic impacts. Previously, lead disrupted cerebellar neurons [1] and hippocampal 

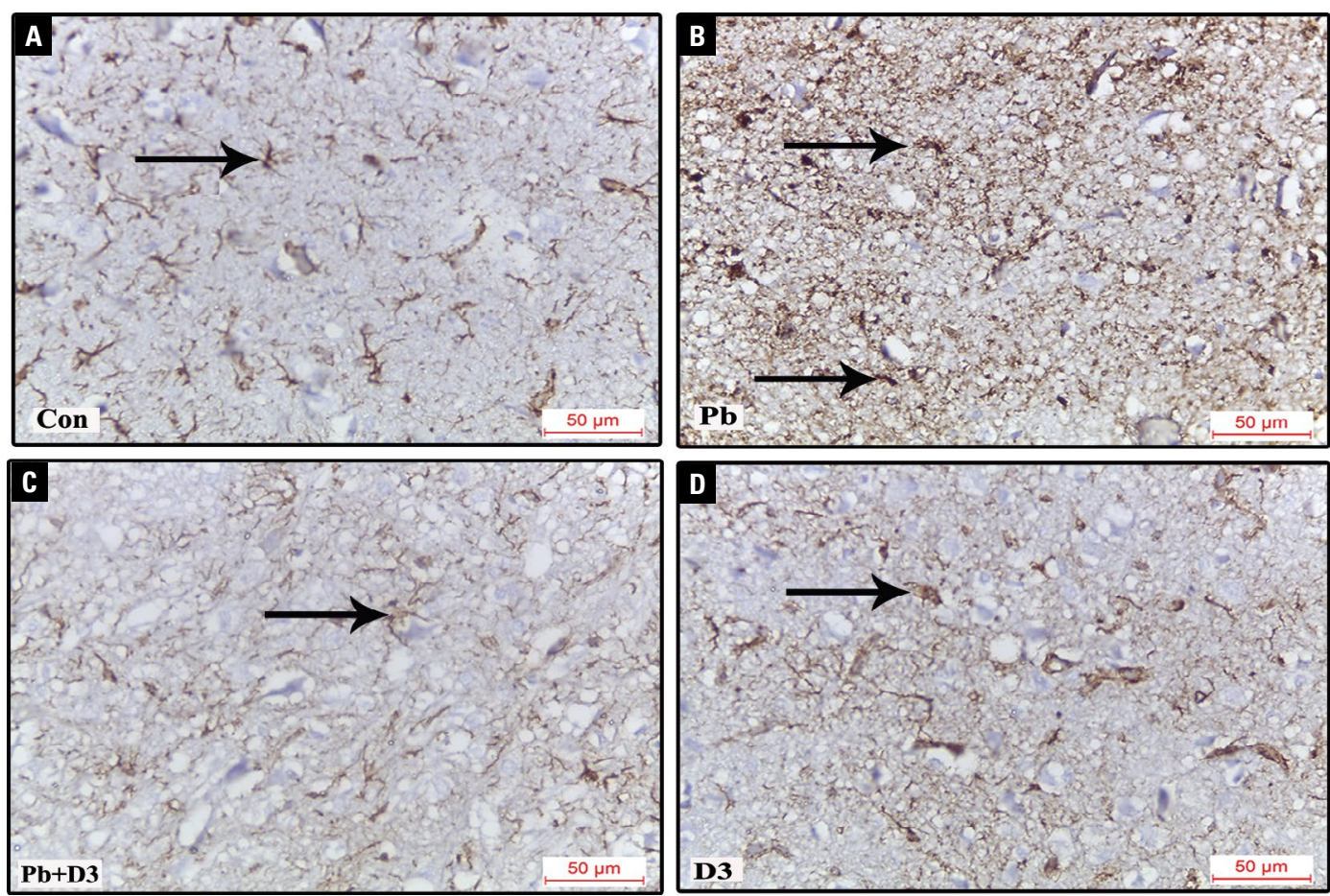

Figure 8. A. The control group (Con) substantia nigra pars compacta (SNpc) appears with a typical glial fibrillatory acid protein (GFAP) reaction (arrow), whereas the lead (Pb) group (B) exhibits increased reaction; C, D. The Pb + D3 and D3 groups, SNpc appear with relatively typical reactions (GFAP $\times 400$, scale bar $=50 \mu \mathrm{m}$ ).
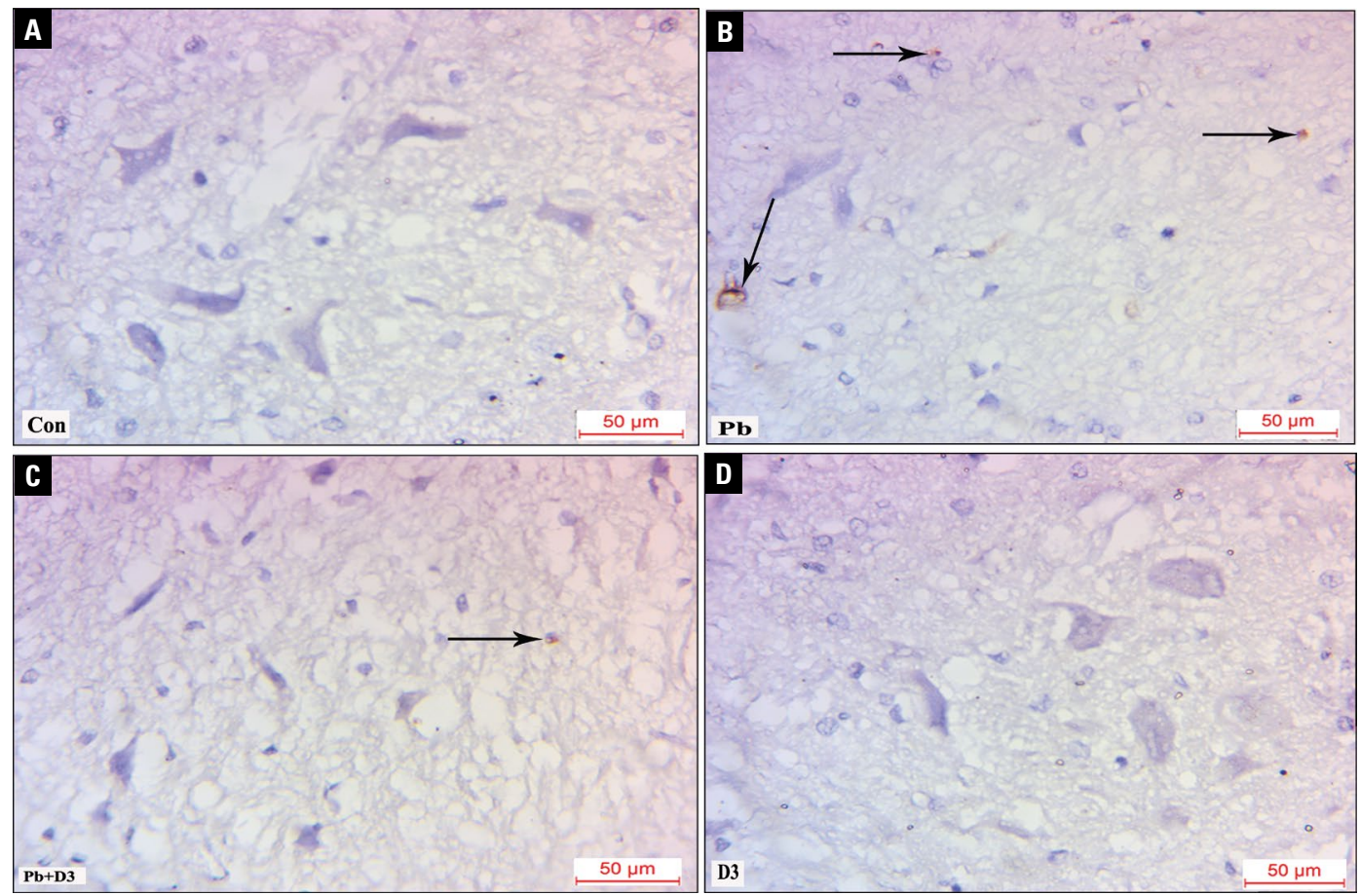

Figure 9. A, B. The control (Con) group substantia nigra pars compacta ( $\mathrm{SNpc}$ ) appears with almost negative caspase-3 immune reaction while the lead $(\mathrm{Pb})$ group SNpc appears with mildly increased reaction (arrows); C, D. The Pb + D3 group SNpc exhibits minimally increased reaction while the D3 group exhibits almost negative reaction (caspase- $3 \times 400$, scale bar $=50 \mu \mathrm{m}$ ).

neurons [20]. Lately, Pb had significantly deteriorated motor functions and coordination in rats. How- ever, L-DOPA administration did not change these findings, supposing that these Parkinsonian-deficits 

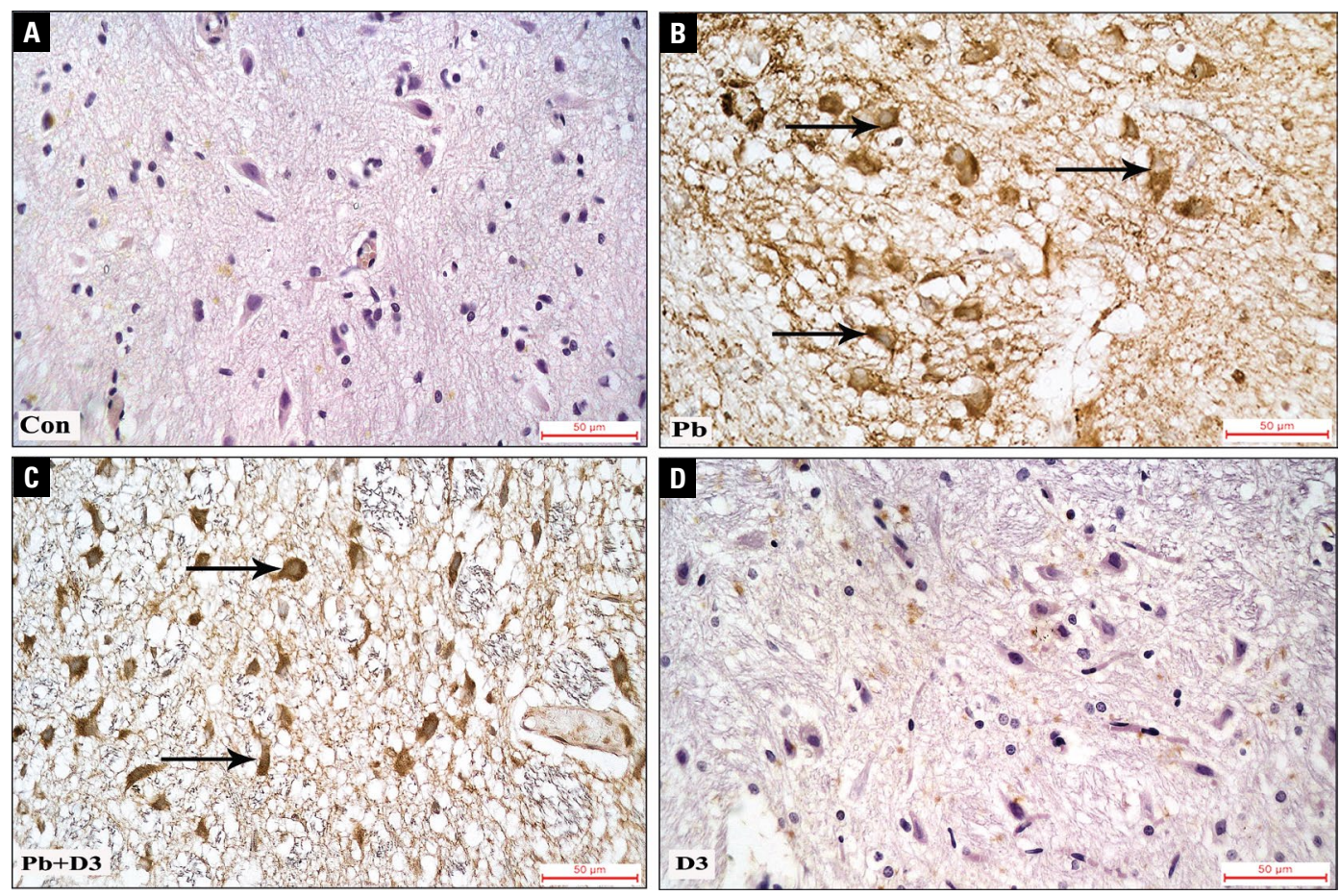

Figure 10. A. The control (Con) group substantia nigra pars compacta ( $\mathrm{SNpc}$ ) with negative $\alpha$-synuclein immune reaction; $\mathbf{B}$. The lead (Pb) group SNpc with markedly increased reaction (arrows); C. The Pb + D3 SNpc with reduced reaction than the Pb group; D. Vitamin D3 group SNpc neurons exhibit scanty $\alpha$-synuclein reaction ( $\alpha$-synuclein $\times 400$, scale bar $=50 \mu \mathrm{m}$ ).

may be due to the decline in noradrenaline but not dopamine [18]. A mild but nonsignificant decrease in nigral-positive Olig-2 expression in rats that received $\mathrm{Pb}$ had been revealed in this study. However. Olig-2 expression significantly increased in the $\mathrm{Pb}+\mathrm{D} 3$ group compared with the control and $\mathrm{Pb}$ groups. Supporting these results, fish oil and omega 3 injections had been proven to promote oligodendrogenesis in a rat model of post-traumatic brain injury associated with the generation of immature neurons and microvessels and significantly improved cognitive recovery [15]. Contradictory to the later findings of this work, aggregates of $\alpha$-synuclein in oligodendrocytes were a characteristic pathological feature of multiple systemic atrophy neurodegenerative disease [10], and neurons to oligodendrocytes transfer of $\alpha$-synuclein had been proven to be the cause of these aggregates [16]. However, the role of oligodendrocytes in lead-induced nigral neurotoxicity is still a candidate for further investigations.

In this study, D3 administration in rats significantly increased their body weight, suggesting its anabolic function, which was a finding that was surprising in the present study. Contradictory to these results, D3 administration in humans during weight loss did not improve weight loss or its related factors [11].
Concomitant D3 administration to lead in group III in this study had significantly improved all nigral biochemical, histological, and immunohistochemical (GFAP, $\alpha$-synuclein, caspase-3, and TH) markers suggesting its possible neuroprotective role in lead-induced nigral neurotoxicity. This neuroprotective role might be mediated through the significant nigral oligodendrogenesis noticed in the rats that received $\mathrm{Pb}+\mathrm{D} 3$. Supporting these results, D3 had ameliorated the oxidative stress markers, upregulated $\mathrm{TH}$ immunoexpression, and attenuated (tumour necrosis factor- $\alpha$ ) immune-reaction in the 6 -hydroxydopamine (6-OHDA) exposed striata [8]. However, Lima et al. [8] reported a decrease in anti-D3 immunostaining in 6-OHDA-exposed striata, which was not found in the lead SNpc group in this study. Also, unilateral intrastriatal injections of 6-OHDA in rats demonstrated declined vitamin $D$ levels in their cardiac tissues [12]. The neuroprotective role of $D 3$ in lead-induced nigral neurotoxicity might be attributed to antioxidative stress mechanisms. Presumably, one of the major causes of neuropathological degeneration in $\mathrm{SNpc}$ neuronal diseases is the cellular biomolecules oxidative stress injury as detected by the markedly increased or decreased body levels of selenium in PD patients [5]. Further studies in humans are still need- 
ed with different doses to match the improvement noticed in animal studies.

\section{CONCLUSIONS}

Lead $(\mathrm{Pb})$ may induce SNpc neurotoxicity presumably through activation of caspase-11 and induction of synucleinopathy. D3 may protect against this neurotoxicity probably through an oligodendrogenic effect.

\section{Conflict of interest: None declared}

\section{REFERENCES}

1. Abubakar K, Muhammad Mailafiya M, Danmaigoro A, et al. Curcumin attenuates lead-induced cerebellar toxicity in rats via chelating activity and inhibition of oxidative stress. Biomolecules. 2019; 9(9), doi: 10.3390/biom9090453, indexed in Pubmed: 31489882.

2. Anderson $\mathrm{FL}$, von Herrmann $\mathrm{KM}$, Andrew $\mathrm{AS}$, et al. Plasma-borne indicators of inflammasome activity in Parkinson's disease patients. NPJ Parkinsons Dis. 2021; 7(1): 2, doi: 10.1038/s41531-020-00147-6, indexed in Pubmed: 33398042.

3. Bancroft JD, Layton C. Connective and other mesenchymal tissues with their stains. In: Suvarna SK, Bancroft JD (eds.). Bancroft's Theory and Practice of Histological Techniques. Eighth Ed. Elsevier Limited 2019: 53-175.

4. BaSalamah MA, Abdelghany AH, El-Boshy M, et al. Vitamin $D$ alleviates lead induced renal and testicular injuries by immunomodulatory and antioxidant mechanisms in rats. Sci Rep. 2018; 8(1): 4853, doi: 10.1038/s41598-01823258-w, indexed in Pubmed: 29556070.

5. Ellwanger JH, Franke SIR, Bordin DL, et al. Biological functions of selenium and its potential influence on Parkinson's disease. An Acad Bras Cienc. 2016; 88(3 Suppl): 1655-1674, doi: 10.1590/0001-3765201620150595, indexed in Pubmed: 27556332.

6. Fullard ME, Duda JE. A review of the relationship between vitamin $\mathrm{d}$ and parkinson disease symptoms. Front Neurol. 2020; 11: 454, doi: 10.3389/fneur.2020.00454, indexed in Pubmed: 32536905.

7. Furuya T, Hayakawa H, Yamada M, et al. Caspase-11 mediates inflammatory dopaminergic cell death in the 1-methyl-4-phenyl-1,2,3,6-tetrahydropyridine mouse model of Parkinson's disease. J Neurosci. 2004; 24(8): 1865-1872, doi: 10.1523/JNEUROSCI.3309-03.2004, indexed in Pubmed: 14985426.

8. Lima LAR, Lopes MJ, Costa RO, et al. Vitamin D protects dopaminergic neurons against neuroinflammation and oxidative stress in hemiparkinsonian rats. J Neuroinflammation. 2018; 15(1): 249, doi: 10.1186/s12974-018-1266-6, indexed in Pubmed: 30170624.

9. Mahul-Mellier AL, Burtscher J, Maharjan N, et al. The process of Lewy body formation, rather than simply $\alpha$-synuclein fibrillization, is one of the major drivers of neurodegeneration.
Proc Natl Acad Sci U S A. 2020; 117(9): 4971-4982, doi: 10.1073/pnas.1913904117, indexed in Pubmed: 32075919.

10. Marmion DJ, Rutkowski AA, Chatterjee D, et al. Viral-based rodent and nonhuman primate models of multiple system atrophy: Fidelity to the human disease. Neurobiol Dis. 2021; 148: 105184, doi: 10.1016/j.nbd.2020.105184, indexed in Pubmed: 33221532.

11. Mason C, Xiao L, Imayama I, et al. Vitamin D3 supplementation during weight loss: a double-blind randomized controlled trial. Am J Clin Nutr. 2014; 99(5): 1015-1025, doi: 10.3945/ajcn.113.073734, indexed in Pubmed: 24622804

12. Nejm MB, Guimarães-Marques MJ, Oliveira LF, et al. Assessment of vitamin $D$ and inflammatory markers profile in cardiac tissue on Parkinson disease animal model. Pharmacol Rep. 2020; 72(2): 296-304, doi: 10.1007/ s43440-020-00074-6, indexed in Pubmed: 32124387.

13. Perry A, Brat DJ. Practical surgical neuropathology: a diagnostic approach. 2nd ed. Chapter 27: Biopsy Pathology of neurodegenerative disorders in adults. Philadelphia, PA 2018.

14. Poewe W, Seppi K, Tanner CM, et al. Parkinson disease. Nat Rev Dis Primers. 2017; 3: 17013, doi: 10.1038/ nrdp.2017.13, indexed in Pubmed: 28332488.

15. $\mathrm{Pu} \mathrm{H}$, Jiang $X$, Wei $Z$, et al. Repetitive and prolonged omega-3 fatty acid treatment after traumatic brain injury enhances long-term tissue restoration and cognitive recovery. Cell Transplant. 2017; 26(4): 555-569, doi: 10.3727/096368916X693842, indexed in Pubmed: 27938482

16. Reyes JF, Rey NL, Bousset L, et al. Alpha-synuclein transfers from neurons to oligodendrocytes. Glia. 2014; 62(3): 387-398, doi: 10.1002/glia.22611, indexed in Pubmed: 24382629.

17. Ross MH, Pawlina W. Histology: a text and atlas - with correlated cell and molecular biology. 7th ed. Chapter 12. Wolters Kluwer Health, Philadelphia 2016: 378.

18. Sabbar M, Delaville $C$, De Deurwaerdère $P$, et al. Lead-Induced atypical parkinsonism in rats: behavioral, electrophysiological, and neurochemical evidence for a role of noradrenaline depletion. Front Neurosci. 2018; 12: 173, doi: 10.3389/fnins.2018.00173, indexed in Pubmed: 29615861.

19. Wang T, Zheng W, Xu He, et al. Clioquinol inhibits zinc-triggered caspase activation in the hippocampal CA1 region of a global ischemic gerbil model. PLoS One. 2010; 5(7): e11888, doi: 10.1371/journal.pone.0011888, indexed in Pubmed: 20686690.

20. Xu J, Yan HC, Yang Bo, et al. Effects of lead exposure on hippocampal metabotropic glutamate receptor subtype 3 and 7 in developmental rats. J Negat Results Biomed. 2009; 8: 5, doi: 10.1186/1477-5751-8-5, indexed in Pubmed: 19374778

21. Yachnis AT, Rivera-Zengotita ML. Neuropathology. Chapter: Arkinson's Disease and Related Alpha-Synucleinopathies. Saunders, an imprint of Elsevier Inc., Philadelphia 2014: 258-264. 